Nicolás Serrano

\title{
Calibration strategies to validate predictive models: is new always better?
}

Received: 2 April 2012

Accepted: 6 April 2012

Published online: 15 May 2012

(C) Copyright jointly held by Springer and ESICM 2012

This editorial refers to the article available at:

doi:10.1007/s00134-012-2578-0.

\section{N. Serrano $(\bullet)$}

Intensive Care Department,

Hospital Universitario de Canarias,

La Laguna, Tenerife, Canary Islands 38320, Spain

e-mail: nicolas.serrano@ doctorserrano.com

Tel.: +34-922-678000

Fax: +34-922-662245

Calibration along with discrimination is an important measure of accuracy to validate predictive logistic regression models. Most predictive models in intensive care such as Simplified Acute Physiology Score (SAPS) II $[1]$ and SAPS $3[2,3]$ consider the binary outcome whether a patient will be alive or dead at hospital discharge. Discrimination measures how well the model can distinguish between patients who die and those who survive. Discrimination is usually assessed by the area under the receiver operating characteristic curve (AU-ROC) [4] This statistic evaluates each pair of observations that have different outcomes and calculates the proportion of times when the patient who died had a higher predicted mortality than did the survivor. The AU-ROC ranges from 0.50 (no discrimination: complete binary random of $50 \%$ similar to flipping a coin) to 1.00 (100\% correct discrimination of the model) [4].

Calibration measures the model's ability to generate predictions that are on average close to the average observed outcome. Calibration has traditionally been approached in two steps. First, to investigate the overall ability to correctly relate the actual occurrence of the event to its estimated probability using statistical methods. The most widely used method is the HosmerLemeshow (H-L) test [5], which examines how well the percentage of observed deaths matches the percentage of predicted deaths over deciles of predicted risk. A $p$ value greater than 0.05 is needed to conclude that there are no significant differences between the observed and expected outcomes and therefore the model has good overall calibration. Second, to localize possible deviations across risk strata by means of calibration plots with observed outcomes versus expected probabilities of mortality. The calibration plot, also named calibration curve, intends to provide complementary information over subsets. If the model calibrates well, there will not be a substantial deviation from the $45^{\circ}$ line of perfect fit or bisector. On the contrary, miscalibration of the model will be a function of expected probability.

The $\mathrm{H}-\mathrm{L}$ test is easy to compute and its interpretation is intuitive, but it has acknowledged limitations such as being very sensitive to sample size [6-8]. The traditional plot or calibration curve also has some disadvantages: first, rather than a curve, it is a jagged line connecting the points in the plot; second, it is not accompanied by any information on the statistical significance of deviations from the bisector [9].

Methods to measure the calibration of predictive models have remained unchanged for a long time, but new insights are now provided. In this issue of Intensive Care Medicine, Poole et al. [10] present a multicenter study aimed at comparing the performance of SAPS II and SAPS 3 in predicting hospital mortality. Discrimination was measured as usual using ROC curves and both models discriminated fairly. Interestingly, the calibration ability of the systems was measured using a new approach named calibration belt [9].

Both systems SAPS II and SAPS 3 calibrated poorly in this study. SAPS II did not show significant deviations 
from ideal calibration in the first two deciles of risk, but it overpredicted mortality in higher-risk classes. SAPS 3 overpredicted mortality homogeneously across all risk classes and showed a higher overprediction rate than SAPS II. Similarly, a common finding in most external validation studies is that models show poor calibration, while discrimination is usually good for all of them [1113]. Miscalibration of SAPS 3 has been already reported in several previous studies [11-17]. Although good performance of SAPS 3 has been shown in some general settings [18, 19], in most instances SAPS 3 presents better calibration in specific subsets of critically ill patients [16, 18, 20-26]. SAPS 3 has been also compared to SAPS II in previous studies using the traditional approach of calibration [14-17, 21, 22, 25-27]. In these studies both SAPS II and SAPS 3 provided excellent discrimination [14-17, 21, 22, 25-27], but also overestimated hospital mortality $[14,28]$ and poor calibration was usually found between them or even being poorer for SAPS 3 [15-17, 27, 28]. Like in the present study, both SAPS II and SAPS 3 have shown adequate inter-rater reliability, but the standardized mortality ratios are still likely to be influenced by the rater's scoring practice [29].

Thus, conclusions of this new study do not substantially differ from previous ones. On the basis of the results of Poole et al., changes over time may be a possible interpretation as to why the older score is not considered better than the more recent one. In this way, continuous improvements in health care possibly have a bigger impact on sicker patients, whereas unchanged conventional treatments have been given to low-risk patients since a long time ago. Although clustering of patients in low-risk classes is unavoidable, about half of the sample in the present study was clustered in low-risk classes. This would mean that SAPS II could calibrate better in this skewed sample of patients. The authors hypothesize that the model used to develop SAPS 3 was not able to adequately take account of factors such as differences in case-mix, influence of unmeasured context-sensitive variables, and the phenomenon of overfitting, but regrettably such questions remain unanswered.

Although Poole et al. focused in this study on the comparison between SAPS II and SAPS 3, in our opinion the main relevance of their work is to introduce the implementation of the calibration belt approach [9] as a new calibration-assessment tool. Calibration of SAPS II and SAPS 3 mortality predictions was measured using the new method. The authors have made an empiric demonstration of this new statistical tool to assess the dependence of calibration on the expected probability by plotting the corresponding curve and its confidence band. The methodology to create a confidence band for the calibration curve is based on a function that relates the expected to observed probabilities across classes of risk. The calibration belt allows one to spot the ranges of risk where there is a significant deviation from the ideal calibration, and the direction of the deviation to be indicated. This method also enables confidence intervals to be computed for the curve, which can be plotted as a calibration belt. This approach allows one to discriminate the ranges in which the model miscalibrates in addition to indicating the direction of this phenomenon [9].

We can argue that the $\mathrm{H}-\mathrm{L}$ statistics associated with the traditional calibration plot would have provided more or less the same information. In fact, such calibration curve plots are based on strata from H-L goodness-of-fit test and these deciles of risk would have also been able to indicate the direction, extent, and risk classes affected by deviations [5-7]. However, we must consider the new approach as a step forward because $\mathrm{H}-\mathrm{L}$ statistics and traditional calibration plot the average risk in each riskclass, whereas the new method is based on a continuous function that does not average the risk in subgroups.

It would have been better if the calibration belt had been tested along with the traditional approach in the present study in order to measure calibration in a simultaneous way. Why not use both the traditional approach and the new one and compare them? We must accept that the mathematical basis of the new approach has been shown elsewhere [9]. On such theoretical grounds, the internal validity is possibly sufficient to grant external validity. Other standard methods and also the $\mathrm{H}-\mathrm{L}$ test were just used because the theoretical basis they were based on clearly indicated their value and their limits [5, 7] and no gold standard exists to be compared with. However, the H-L test was compared to similar ones via simulation under both null and alternative scenarios after their development [7] and also tested again more recently in the same way [6]. Such stress tests have given us new insights into the limitations of the $\mathrm{H}-\mathrm{L}$ test $[6,7]$.

Thus, although currently we acknowledge limitations in $\mathrm{H}-\mathrm{L}$ tests and the traditional plot methods, as yet we have no indication of the potential limitations of the new calibration belt approach. Although the new method may offer a more analytical view and a substantial improvement in the assessment of quality of care compared to other available tools, its sampling characteristics have not been explored as well as its confidence intervals. Since it is unlikely that any model in practice can be perfectly calibrated, it is important that the interpretation of accuracy considers the sample size. Although the method is possibly accurate on theoretical grounds, future investigations to determine its theoretical limits are warranted.

In summary, models that predict mortality for patients admitted to ICUs have traditionally relied on the $\mathrm{H}-\mathrm{L}$ test as a determinant of model calibration. As a result, an important amount of previous research has been done using this approach. When such methods have been applied to comparisons between SAPS II and SAPS 3, results of these previous studies do not substantially differ from the present one, and they reveal miscalibration of SAPS 3 as the true problem. The use of the calibration 
belt is an important methodological contribution of the tool is mandatory in order to determine potential limitapresent study but the assessment of this new statistical tions as well as new additional advantages.

\section{References}

1. Le Gall JR, Lemeshow S, Saulnier F (1993) A new Simplified Acute Physiology Score (SAPS II) based on a European/North American multicenter study. JAMA 270:2957-2963

2. Metnitz PG, Moreno RP, Almeida E, Jordan B, Bauer P, Campos RA, Iapichino G, Edbrooke D, Capuzzo M, Le Gall JR (2005) SAPS 3-from evaluation of the patient to evaluation of the intensive care unit. Part 1: objectives, methods and cohort description. Intensive Care Med 31:1336-1344

3. Moreno RP, Metnitz PG, Almeida E, Jordan B, Bauer P, Campos RA, Iapichino G, Edbrooke D, Capuzzo M, Le Gall JR (2005) SAPS 3-from evaluation of the patient to evaluation of the intensive care unit. Part 2: development of a prognostic model for hospital mortality at ICU admission. Intensive Care Med 31:1345-1355

4. Hanley JA, McNeil BJ (1982) The meaning and use of the area under a receiver operating characteristic (ROC) curve. Radiology 143:29-36

5. Hosmer DW, Lemeshow S (2000) Applied logistic regression, 2nd edn. Wiley, New York

6. Kramer AA, Zimmerman JE (2007) Assessing the calibration of mortality benchmarks in critical care: the Hosmer-Lemeshow test revisited. Crit Care Med 35:2052-2056

7. Hosmer DW, Hosmer T, Le CS, Lemeshow S (1997) A comparison of goodness-of-fit tests for the logistic regression model. Stat Med 16:965-980

8. Lemeshow S, Teres D, Klar J, Avrunin JS, Gehlbach SH, Rapoport J (1993) Mortality probability models (MPM II) based on an international cohort of intensive care unit patients. JAMA 270:2478-2486

9. Finazzi S, Poole D, Luciani D, Cogo PE, Bertolini G (2011) Calibration belt for quality-of-care assessment based on dichotomous outcomes. PLoS One 6:e16110

10. Poole D, Rossi C, Latronico N, Rossi G, Finazzi S, Bertolini G (2012) Comparison between SAPS II and SAPS 3 in predicting hospital mortality in a cohort of 103 Italian ICUs. Is new always better? Intensive Care Med. doi:10.1007/s00134-012-2578-0
11. Nassar AP Jr, Mocelin AO, Nunes AL Giannini FP, Brauer L, Andrade FM, Dias CA (2011) Caution when using prognostic models: A prospective comparison of 3 recent prognostic models. J Crit Care. doi: 10.1016/j.jcrc.2011.08.016

12. Khwannimit $B$, Bhurayanontachai $R$ (2010) The performance and customization of SAPS 3 admission score in a Thai medical intensive care unit. Intensive Care Med 36:342-346

13. Poole D, Rossi C, Anghileri A, Giardino M, Latronico N, Radrizzani D, Langer M, Bertolini G (2009) External validation of the Simplified Acute Physiology Score (SAPS) 3 in a cohort of 28,357 patients from 147 Italian intensive care units. Intensive Care Med 35:1916-1924

14. Khwannimit B, Bhurayanontachai R (2011) A comparison of the performance of Simplified Acute Physiology Score 3 with old standard severity scores and customized scores in a mixed medical-coronary care unit. Minerva Anestesiol 77:305-312

15. Capuzzo M, Scaramuzza A, Vaccarini B, Gilli G, Zannoli S, Farabegoli L, Felisatti G, Davanzo E, Alvisi R (2009) Validation of SAPS 3 admission score and comparison with SAPS II. Acta Anaesthesiol Scand 53:589-594

16. Sakr Y, Krauss C, Amaral AC, ReaNeto A, Specht M, Reinhart K, Marx G (2008) Comparison of the performance of SAPS II, SAPS 3, APACHE II, and their customized prognostic models in a surgical intensive care unit. $\mathrm{Br} \mathbf{J}$ Anaesth 101:798-803

17. Ledoux D, Canivet JL, Preiser JC, Lefrancq J, Damas P (2008) SAPS 3 admission score: an external validation in a general intensive care population. Intensive Care Med 34:1873-1877

18. Silva Junior JM, Malbouisson LM, Nuevo HL, Barbosa LG, Marubayashi LY, Teixeira IC, Nassar Junior AP, Carmona MJ, Silva IF, Auler Junior JO, Rezende E (2010) Applicability of the Simplified Acute Physiology Score (SAPS 3) in Brazilian hospitals. Rev Bras Anestesiol 60:20-31

19. Mbongo CL, Monedero P, GuillenGrima F, Yepes MJ, Vives M, Echarri G (2009) Performance of SAPS 3, compared with APACHE II and SOFA, to predict hospital mortality in a general ICU in Southern Europe. Eur J Anaesthesiol 26:940-945
20. Costa e Silva VT, de Castro I, Liano F, Muriel A, Rodriguez-Palomares JR, Yu L (2011) Performance of the thirdgeneration models of severity scoring systems (APACHE IV, SAPS 3 and MPM-III) in acute kidney injury critically ill patients. Nephrol Dial Transplant 26:3894-3901

21. Lim SY, Ham CR, Park SY, Kim S, Park MR, Jeon K, Um SW, Chung MP, Kim H, Kwon OJ, Suh GY (2011) Validation of the Simplified Acute Physiology Score 3 scoring system in a Korean intensive care unit. Yonsei Med J 52:59-64

22. Soares M, Silva UV, Teles JM, Silva E, Caruso P, Lobo SM, Dal PF, Azevedo LP, de Carvalho FB, Salluh JI (2010) Validation of four prognostic scores in patients with cancer admitted to Brazilian intensive care units: results from a prospective multicenter study. Intensive Care Med 36:1188-1195

23. Maccariello E, Valente C, Nogueira L, Bonomo $\mathrm{H}$, Ismael $\mathrm{M}$, Machado JE, Baldotto F, Godinho M, Valenca R, Rocha E, Soares M (2010) SAPS 3 scores at the start of renal replacement therapy predict mortality in critically ill patients with acute kidney injury. Kidney Int 77:51-56

24. Metnitz B, Schaden E, Moreno R, Le Gall JR, Bauer P, Metnitz PG (2009) Austrian validation and customization of the SAPS 3 admission score. Intensive Care Med 35:616-622

25. Tsai CW, Lin YF, Wu VC, Chu TS, Chen YM, Hu FC, Wu KD, Ko WJ (2008) SAPS 3 at dialysis commencement is predictive of hospital mortality in patients supported by extracorporeal membrane oxygenation and acute dialysis. Eur J Cardiothorac Surg 34:1158-1164

26. Soares M, Salluh JI (2006) Validation of the SAPS 3 admission prognostic model in patients with cancer in need of intensive care. Intensive Care Med 32:1839-1844

27. Strand K, Soreide E, Aardal S, Flaatten H (2009) A comparison of SAPS II and SAPS 3 in a Norwegian intensive care unit population. Acta Anaesthesiol Scand 53:595-600

28. Zajac K, Andres J, Zajac M (2009) A comparison of SAPS 2 and SAPS 3. Acta Anaesthesiol Scand 53:1230-1231

29. Strand K, Strand LI, Flaatten H (2010) The interrater reliability of SAPS II and SAPS 3. Intensive Care Med 36:850-853 